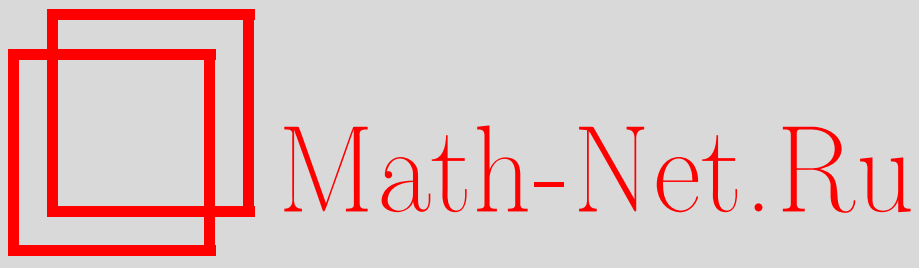

А. И. Данилушкин, О. О. Осипов, И. В. Фрыгин, Аналитическая модель процесса непрерывного индукционного нагрева заготовок подшипниковых колец, Вестн. Сам. гос. техн. ун-та. Сер. Физ.-мат. науки, 2000, выпуск 9, 200-203

DOI: https://doi.org/10.14498/vsgtu53

Использование Общероссийского математического портала Math-Net.Ru подразумевает, что вы прочитали и согласны с пользовательским соглашением

http://www.mathnet.ru/rus/agreement

Параметры загрузки:

IP : 54.81 .137 .203

26 апреля 2023 г., 15:12:27

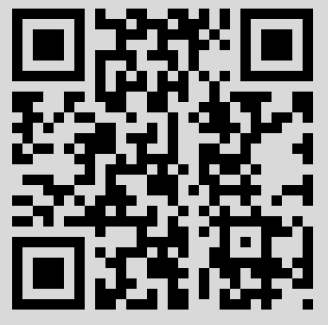


6. Борисов А.Н., Алексеев А.В., Меркурьева Г.В. Обработка нечетной информации в системах принятия решений. М.: Радио и связь, 1989. 303 с.

7. Гревцев О.И. Нахождение оптимального решения многокритериальных задач // Вестн. Сам ГТУ. Сер. «Физ.-мат. науки». Вып. 6. Самара: СамГТУ, 1998. С. 135-136.

8. Гревцев О.И. Выбор оптимальных решений многокритериальных задач // Математическое моделирование и краевые задачи: Тр. девятой межвуз. конф. Самара: СамГТУ, 1999. С.31-33.

УДК $62-50$

А.И. Данилушкин, О.О. Осипов, И.В. Фрыгин

\section{АНАЛИТИЧЕСКАЯ МОДЕЛЬ ПРОЦЕССА НЕПРЕРЫВНОГО ИНДУКЦИОННОГО НАГРЕВА ЗАГОТОВОК ПОДШИПНИКОВЫХ КОЛЕЦ}

Предложена аналитическая модель проиесса непрерывного индукиионного нагрева кольцевых заготовок, учитывающая тепловые потери с внутренней поверхности и ориентированная на реше-

ние задач оптимального проектирования и управления.

Индукционные нагревательные установки непрерывного действия являются необходимой составной частью крупных технологических комплексов по обработке металлов давлением, например, при прессовании, штамповке, раскатке, калибровке и т.д.

В индукторе непрерывного действия располагается одновременно несколько заготовок одной партии, которые нагреваются в процессе их равномерного перемещения от входа к выходу. Темп выдачи заготовок задается деформирующим оборудованием.

Процесс непрерывного индукционного нагрева колец описывается нестационарным уравнением теплопроводности в цилиндрической системе координат [1]

$$
\frac{\partial \theta(l, v, \varphi)}{\partial \varphi}=\frac{\partial^{2} \theta(l, v, \varphi)}{\partial l^{2}}+\frac{1}{l} \cdot \frac{\partial \theta(l, v, \varphi)}{\partial l}-\beta(y) \frac{\partial \theta(l, v, \varphi)}{\partial v}+\Phi(l, v, \varphi) .
$$

Уравнение (1) дополняется начальными

$$
\theta(l, v, 0)=\theta_{0}(l, v)
$$

и граничными условиями второго рода

$$
\begin{gathered}
\frac{\partial \theta(1, v, \varphi)}{\partial l}=q_{1}(1, v, \varphi), \\
\frac{\partial \theta\left(l_{1}, v, \varphi\right)}{\partial l}=q_{2}\left(l_{1}, v, \varphi\right) ; \\
\theta(l, 0, \varphi)=\theta_{0} .
\end{gathered}
$$

Здесь относительные значения $\theta(l, v, \varphi), q_{1}(v, \varphi), q_{2}(l, \varphi)$ соответственно для температурного поля $T(r, x, t)$ полого цилиндра, потоков тепла $Q_{1}(R, t, x), Q_{2}\left(R_{1}, x, t\right)$ с внешней и внутренней поверхностей цилиндра соответственно, а также относительные значения $\Phi(l, v, \varphi)$ источников $\Xi(r, x, t)$ внутреннего тепловыделения вводятся согласно соотношениям [2]

$$
\left.\begin{array}{l}
\theta(l, v, \varphi)=\frac{\lambda T(r, x, t)}{P_{\max } \cdot R_{1}{ }^{2}} ; \\
q_{1}(\nu, \varphi)=\frac{Q_{1}\left(R_{1}, t, x\right)}{P_{\max } \cdot R_{1}{ }^{2}} ; q_{2}(l, \varphi)=\frac{Q_{2}\left(R_{2}, t, x\right)}{P_{\max } \cdot R_{1}{ }^{2}} ; \\
\Phi(l, v, \varphi)=\frac{\Xi(r, x, t)}{P_{\max }} ; \\
l=\frac{r}{R} ; v=\frac{x}{L} ; \varphi=\frac{a t}{R_{2}{ }^{2}} ; \alpha(y)=\frac{\lambda_{*}}{\lambda(y)} ; \beta(y)=\frac{V \cdot R_{1}^{2}}{a \cdot L} .
\end{array}\right\}
$$


Действительный характер зависимости объемного распределения внутренних источников тепла от температуры для практических расчетов с приемлемой точностью можно заменить зависимостью их от пространственных координат $r$ и $x$.

Возможность такой замены появляется благодаря тому, что у нагревателя непрерывного действия, внутри которого заготовки перемещаются вдоль продольной оси, функция распределения мощности внутренних теплоисточников по длине имеет ступенчатый характер, обусловленный нелинейной зависимостью магнитной проницаемости от температуры [3]. В соответствии с этим нагреватель условно разделен на 3 зоны, в пределах каждой из которых теплофизические характеристики считают постоянными. Так как в установившемся режиме температура изделия однозначно связана с продольной координатой нагревателя, функция $F(x)$ распределения мощности внутреннего тепловыделения по длине может быть представлена в виде [4]

$$
F(x)=\left\{\begin{array}{l}
F_{1}, \forall x \in\left[0, x_{1}\right] \\
F_{2}, \forall x \in\left[x_{1}, x_{2}\right], \\
F_{3}, \forall x \in\left[x_{2}, L\right]
\end{array}\right.
$$

где $x_{1}$ и $x_{2}$-границы участков индуктора, в пределах которых теплофизические свойства и функции внутреннего тепловыделения считаются постоянными. Указанные координатные зависимости в установившемся режиме легко определяются из решения уравнения теплопроводности.

Управление установками непрерывного действия сводится к решению двух проблем:

управление пространственным распределением мощности внутреннего тепловыделения по длине индуктора в стационарном режиме работы;

управление нестационарными режимами работы нагревателя при изменении производительности, пуске, смене номенклатуры и т. д.

Задача оптимизации установившихся режимов работы индукционных нагревателей в технологической линии с деформирующим оборудованием сводится к определению оптимального распределения мощности источников внутреннего тепловыделения по длине индукционной системы, которое обеспечивает в условиях заданных ограничений получение требуемой точности температурного распределения по объему нагреваемых изделий на выходе из нагревателя. Для установившихся режимов работы индукционного нагревателя в технологической линии с деформирующим оборудованием распределение температуры при нагреве изделий с постоянной скоростью перемещения и постоянной удельной мощностью описывается уравнением стационарной теплопроводности вида

$$
\frac{\partial^{2} \theta(l, v)}{\partial^{2}}+\frac{1}{l} \cdot \frac{\partial \theta(l, v)}{\partial}-\beta(v) \frac{\partial \theta(l, v)}{\partial v}+Q(l, v)=0
$$

с граничными условиями

$$
\frac{\partial \theta(1, v)}{\partial l}=q_{1}(1, v), \frac{\partial \theta\left(l_{1}, v\right)}{\partial l}=q_{2}\left(l_{1}, v\right), \theta(l, 0)=\theta_{c} .
$$

Для решения уравнения (8) с граничными условиями (9) применим конечное интегральное преобразование Ханкеля по радиальной координате $l$ вида

$$
\bar{f}\left(\mu_{\kappa}\right)=\int_{l_{1}}^{l_{2}} l \psi_{k}\left(\mu_{k} l\right) f(l) d l,
$$

где ядро преобразования $\psi_{k}\left(\mu_{k} l\right)$ определяется, согласно [5], как решение граничной задачи Штурма- Лиувилля в форме

$$
\psi_{k}\left(\mu_{k} l\right)=Y_{1}\left(\mu_{k}\right) I_{0}\left(\mu_{k} l\right)-I_{1}\left(\mu_{k}\right) Y_{0}\left(\mu_{k} l\right) .
$$

Здесь $Y_{1}\left(\mu_{k}\right), I_{0}\left(\mu_{k} l\right), I_{1}\left(\mu_{k}\right), Y_{0}\left(\mu_{k} l\right)-$ функции Бесселя; $\mu_{k}$ - собственные числа, определяемые как корни уравнения

$$
Y_{1}(\mu) I_{1}\left(\mu l_{1}\right)-I_{1}(\mu) Y_{1}\left(\mu l_{1}\right)=0 .
$$

Применяя интегральное преобразование (10) с ядром (11) к уравнению (8) и граничным условиям (9), получим бесконечную систему обыкновенных дифференциальных уравнений первого порядка относительно изображения $\bar{\theta}\left(\mu_{k}, l\right)$ по Ханкелю температурного распределения 
$\beta(y) \frac{d \bar{\theta}\left(\mu_{k}, v\right)}{d v}+\mu_{k}^{2} \bar{\theta}\left(\mu_{k}, v\right)=Q\left(\mu_{k}, v\right) G(y) \alpha(y)-q_{1}(1, v) \psi_{k}\left(\mu_{k}\right)+q_{2}\left(l_{1}, v\right) \psi_{k}\left(\mu_{k} l_{1}\right)$

с граничным условием

$$
\bar{\theta}\left(\mu_{k}, 0\right)=\int_{l_{1}}^{1} l \theta_{c} \psi\left(\mu_{k} l\right) d l
$$

Здесь $\bar{\theta}\left(\mu_{k}, v\right)=\int_{l_{1}}^{1} l \theta(l, v) \psi_{k}\left(\mu_{k} l\right) d l ; \bar{Q}\left(\mu_{k}, v\right)=\int_{l_{1}}^{1} l Q(l, v) \psi_{k}\left(\mu_{k} l\right) d l$.

Решение уравнения (13) имеет вид

$$
\bar{\theta}\left(\mu_{k}, v\right)=e^{-\int_{0}^{v} \frac{\mu_{k}^{2}}{\beta(z)} d z}\left\{\bar{\theta}\left(\mu_{k}, 0\right)+\int_{0}^{v}\left[\frac{\alpha(x) Q\left(\mu_{k} x\right) G(x)}{\beta(x)}+\frac{\bar{q}(x)}{\beta(x)} \psi_{k}\left(\mu_{k}\right)\right] e^{\int \frac{\mu_{k}^{2}}{\beta(z)} d z} d x\right\},
$$

где $\bar{q}(x)=\overline{q_{1}}(1, x)-\bar{q}_{2}\left(l_{1}, x\right) ; \bar{q}_{1}(1, x)=\overline{q_{1}}(1, x) \psi_{k}\left(\mu_{k}\right) ; \bar{q}_{2}\left(l_{1}, x\right)=\overline{q_{2}}\left(l_{1}, x\right) \psi_{k}\left(\mu_{k}, l_{1}\right)$.

Для перехода к оригиналу воспользуемся обратным преобразованием Ханкеля [5].

Решение уравнения (8 ) в окончательной форме принимает вид

$$
\begin{aligned}
& \theta(l, v)=\sum_{k=1}^{\infty} \frac{\psi_{k}\left(\mu_{k} l\right) \pi^{2} \mu_{k}^{2} I_{1}^{2}\left(\mu_{k} l\right)}{2 R_{1}^{2}\left[I_{1}\left(\mu_{k}\right)-I_{1}^{2}\left(\mu_{k} l\right)\right]}\left\{e ^ { - \int _ { 0 } ^ { v } \frac { \mu _ { k } ^ { 2 } } { \beta ( z ) } d z } \left[\int_{l_{1}}^{1} l \theta_{c} \psi_{k}\left(\mu_{k}\right) d l+\int_{0}^{v} \frac{\bar{q}(x)}{\beta(x)} \psi_{k}\left(\mu_{k}\right) e^{\int_{0}^{x} \frac{\mu_{k}^{2}}{\beta(z)} d z} d x+\right.\right. \\
& \left.\left.+\int_{0}^{1} \int_{l_{1}}^{1} \frac{Q(l, v) l \psi_{k}\left(\mu_{k}\right) G(v) \alpha(v)}{\beta(x)} e^{\int_{0}^{v} \frac{\mu_{k}^{2}}{\beta(z)} d z} d x d l\right]\right\}, l \in\left[l_{1}, 1\right], \quad v \in[0,1] .
\end{aligned}
$$

Принятая в форме (7) аппроксимация распределения внутренних источников тепла позволяет для односекционного нагревателя непрерывного и полунепрерывного действия рассматривать процесс нагрева как трехстадийный нагрев, при котором теплофизические параметры $\alpha(v), \quad \beta(v)$, тепловые потери $q(v)$, относительная мощность $G(v)$, функция $Q(l, v)$ распределения внутренних источников тепла принимаются постоянными в пределах каждого участка.

Подставляя последовательно в решение уравнения теплопроводности в форме (16) конкретные выражения соответствующих параметров для каждого участка постоянства, получим после интегрирования и несложных преобразований температурное распределение в загрузке односекционного нагревателя на «холодном», «промежуточном» и «горячем»режимах нагрева соответственно:

$$
\theta_{\text {хол }}(l, v)=\theta_{\text {ср.хол. }}(v)+\sum \Phi\left(\mu_{k}, l\right)\left\{\left[\frac{\bar{Q}_{\text {хол }}\left(\mu_{\pi}\right) G_{\text {Хол }} \alpha_{\text {Хол }}}{\mu_{\pi}^{2}}+\frac{\bar{q}_{\text {хол }} \psi_{k}\left(\mu_{k}\right)}{\mu_{k}^{2}}\right]\left(1-e^{-\frac{\mu_{k}^{2}}{\beta_{\text {хол }}}}\right)\right\},
$$

$\forall v \in\left[0, v_{n \mathrm{p}}\right], \quad \forall l \in\left[l_{1}, 1\right]$

где $\theta_{\text {ср.хол. }}(l, v)=\frac{2}{1-l_{1}^{2}}\left[\frac{q_{\text {хол }}}{\beta_{\text {хол }}} v+\frac{G_{\text {ХОЛ }} \alpha_{\text {ХОЛ }}}{\beta_{\text {Хол }}} v+\theta_{0}(l)\right] ; \bar{Q}_{\text {хол }}\left(\mu_{\kappa}\right)=\int_{l_{1}}^{1} l Q_{\text {хол }}\left(l, \xi_{\text {хол }}\right) \psi_{л}\left(\mu_{л} l\right) d l$.

На «промежуточном» участке

$$
\begin{aligned}
& \theta_{n \mathrm{p}}(l, v)=\theta_{c \mathrm{p} . n \mathrm{p} .}(v)+\sum_{n=1}^{\infty} \Phi\left(\mu_{k}, l\right)\left\{\left[\frac{\bar{Q}_{x o n}\left(\mu_{k}\right) G_{x o l} \alpha_{x o r}}{\mu_{k}^{2}}+\frac{\bar{q}_{x o n} \psi\left(\mu_{k}\right)}{\mu_{k}^{2}}\right]\left(e^{-\frac{\mu_{k}^{2}}{\beta_{x o r}} v-v_{n \mathrm{p}}}-e^{-v_{n \mathrm{p}}}\right)+\right. \\
& \left.+\left[\frac{\bar{Q}_{n \mathrm{p}}\left(\mu_{k}\right) G_{n \mathrm{p}} \alpha_{n \mathrm{p}}}{\mu_{k}^{2}}+\frac{\bar{q}_{n \mathrm{p}} \psi_{n}\left(\mu_{n}\right)}{\mu_{l}^{2}}\right]\left(1-e^{-\frac{\mu_{k}^{2}}{\beta_{n \mathrm{p}}}\left(v-v_{n \mathrm{p}}\right)}\right)\right\}, \forall v \in\left[v_{n \mathrm{p}}, v_{z o \mathrm{p}}\right], \quad \forall l \in\left[l_{1}, 1\right] .
\end{aligned}
$$

Здесь 


$$
\begin{aligned}
& \theta_{c \mathrm{p} . n \mathrm{p}}(l, v)=\frac{2}{1-l_{1}^{2}}\left[\frac{q_{x o n}}{\beta_{x o n}} v_{n \mathrm{p}}+\frac{G_{x o n} \alpha_{x o n}}{\beta_{x o n}} v_{n \mathrm{p}}+\theta_{0}(l)+\frac{q_{n \mathrm{p}}}{\beta_{n \mathrm{p}}}\left(v-v_{n \mathrm{p}}\right)+\frac{G_{n \mathrm{p}} \alpha_{n \mathrm{p}}}{\beta_{n \mathrm{p}}}\left(v-v_{n \mathrm{p}}\right)\right] ; \\
& \bar{Q}_{n \mathrm{p}}\left(\mu_{\kappa}\right)=\int_{l_{1}}^{1} l Q_{n \mathrm{p}}\left(l, \xi_{n \mathrm{p}}\right) \psi_{n}\left(\mu_{n} l\right) d l, \quad v_{k}=\frac{\mu_{k}^{2}}{\beta_{n \mathrm{p}}}\left(v-v_{n \mathrm{p}}\right)+\frac{\mu_{l}^{2}}{\beta_{x o n}} v_{n \mathrm{p}}
\end{aligned}
$$

На «горячем» участке

$$
\begin{aligned}
& \theta_{n \mathrm{p}}(l, v)=\theta_{c \mathrm{p} . \text { гор. }}(v)+\sum_{n=1}^{\infty} \Phi\left(\mu_{k}, l\right)\left\{\left[\frac{\bar{Q}_{\text {хол }}\left(\mu_{k}\right) G_{x о л} \alpha_{\text {xол }}}{\mu_{k}^{2}}+\frac{\bar{q}_{x о л} \psi\left(\mu_{k}\right)}{\mu_{k}^{2}}\right]\left(e^{-\frac{\mu_{k}^{2}}{\beta_{x o n}} v-v_{n \mathrm{p}}}-e^{-v_{n \mathrm{p}}}\right)+\right. \\
& \left.+\left[\frac{\bar{Q}_{n \mathrm{p}}\left(\mu_{k}\right) G_{n \mathrm{p}} \alpha_{n \mathrm{p}}}{\mu_{k}^{2}}+\frac{\bar{q}_{n \mathrm{p}} \psi_{n}\left(\mu_{n}\right)}{\mu_{n}^{2}}\right]\left(e^{\frac{\mu_{n}^{2}}{\beta_{x o l}} v_{n \mathrm{p}} \frac{\mu_{k}^{2}}{\beta_{n \mathrm{p}}}\left(v_{z o \mathrm{p}}-v_{n \mathrm{p}}\right)}-e^{-\frac{\mu_{k}^{2}}{\beta_{n \mathrm{p}}}\left(v-v_{n \mathrm{p}}\right)}\right)\right\}+
\end{aligned}
$$

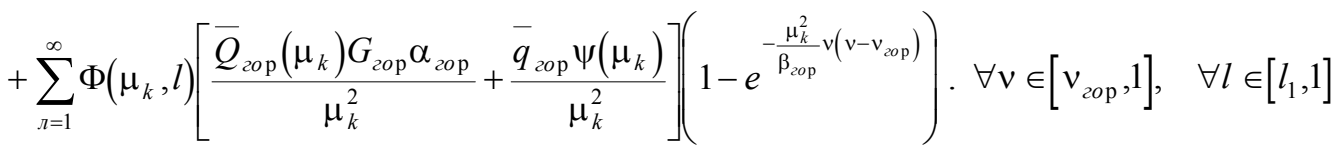

$$
\begin{aligned}
& \text { Здесь } \theta_{\text {ср.гор }}(l, v)=\frac{2}{1-l_{1}^{2}}\left[\frac{q_{x о л}}{\beta_{\text {хол }}} v_{n \mathrm{p}}+\frac{G_{x о л} \alpha_{x о л}}{\beta_{\text {хол }}} v_{n \mathrm{p}}+\theta_{0}(l)+\frac{q_{n \mathrm{p}}}{\beta_{n \mathrm{p}}}\left(v-v_{n \mathrm{p}}\right)+\frac{G_{n \mathrm{p}} \alpha_{n \mathrm{p}}}{\beta_{n \mathrm{p}}}\left(v-v_{n \mathrm{p}}\right)\right]+ \\
& +\frac{q_{\text {гор }}}{\beta_{\text {гор }}}\left(v-v_{\text {гор }}\right)+\frac{G_{\text {гор }} \alpha_{\text {гор }}}{\beta_{x о л}}\left(v-v_{\text {гор }}\right), \quad \bar{Q}_{\text {гор }}\left(\mu_{\kappa}\right)=\int_{l_{1}}^{1} l Q_{\text {гор }}\left(l, \xi_{\text {гор }}\right) \psi_{l}\left(\mu_{l} l\right) d l ; \\
& v_{\text {гор }}=\frac{\mu_{k}^{2}}{\beta_{\text {гор }}}\left(v_{\text {гор }}-v_{n \mathrm{p}}\right)+\frac{\mu_{k}^{2}}{\beta_{n \mathrm{p}}} v_{n \mathrm{p}}+\frac{\mu_{k}^{2}}{\beta_{x о л}} v_{x о л} .
\end{aligned}
$$

На основании полученных выражений выполнен анализ температурных полей кольцевых заготовок с учетом тепловых потерь с внутренней стенки кольца. Показано, что радиальный перепад температур существенно зависит от соотношения толщины стенки кольца и глубины проникновения тока. На «холодном» и «промежуточном» участках индуктора, где глубина проникновения тока значительно меньше толщины стенки кольца, а тепловые потери незначительны, имеет место положительный градиент температуры от внутренней стенки к внешней. На «горячем» участке индуктора глубина проникновения тока существенно увеличивается , что может привести к появлению отрицательного перепада температур. Вероятность такой ситуации увеличивается при уменьшении толщины кольца. Отрицательный перепад температур неблагоприятно отражается на процессе деформации, так как снижает пластичность материала заготовки. Это обстоятельство необходимо учитывать при выборе частоты источника питания.

Предложенная модель процесса индукционного нагрева кольцевых заготовок, учитывающая тепловые потери с внутренней поверхности, рассматривается далее в качестве исходной аналитической модели при решении задач оптимального проектирования индукционных кузнечных нагревателей непрерывного или методического действия.

\section{БИБЛИОГРАФИЧЕСКИЙ СПИСОК}

1. Павлов Н.А. Инженерные тепловые расчеты индукционных нагревателей. М.-Л.: Энергия, 1978. 120 c.

2. Лыков А.В. Тепломассообмен: Справочник. М.: Энергия, 1978. 480 с.

3. Немков В.С., Демидович В.Б. Теория и расчет устройств индукционного нагрева. Л.: Энергоатомиздат, 1988. 280c.

4. Слухоикий А.Е., Рыскин С.Е. Индукторы для индукционного нагрева машиностроительных деталей. Л.: Энергия, 1975. $183 \mathrm{c}$

5. Карташов Э.М. Аналитические методы в теории теплопроводности твердых тел: М.: Высш. школа, 1985. 480 с. 